\title{
Scale-similar clustering of heavy particles in the inertial range of turbulence
}

\author{
Taketo Ariki, ${ }^{1, *}$ Kyo Yoshida ${ }^{2, \dagger}$ Keigo Matsuda, ${ }^{3, \ddagger}$ and Katsunori Yoshimatsu ${ }^{1,8}$ \\ ${ }^{1}$ Institute of Materials and Systems for Sustainability, Nagoya University, Furo-cho, Chikusa-ku, Nagoya 464-8601, Japan \\ ${ }^{2}$ Division of Physics, Faculty of Pure and Applied Sciences, University of Tsukuba, 1-1-1 Tennoudai, Tsukuba 305-8571, Japan \\ ${ }^{3}$ Center for Earth Information Science and Technology, Japan Agency for Marine-Earth Science and Technology, 3173-25 Showa-machi, \\ Kanazawa-ku, Yokohama 236-0001, Japan
}

(Received 25 July 2017; published 22 March 2018)

\begin{abstract}
Heavy particle clustering in turbulence is discussed from both phenomenological and analytical points of view, where the $-4 / 3$ power law of the pair-correlation function is obtained in the inertial range. A closure theory explains the power law in terms of the balance between turbulence mixing and preferential-concentration mechanism. The obtained $-4 / 3$ power law is supported by a direct numerical simulation of particle-laden turbulence.
\end{abstract}

DOI: 10.1103/PhysRevE.97.033109

\section{INTRODUCTION}

Inertial heavy particles, the density of which is sufficiently larger than the fluid density, in turbulent flows are frequently observed (e.g., cloud droplets in the atmosphere [1] or space dusts in the accretion disks [2,3]), and form a nonuniform spatial distribution, which is referred to as turbulent particle clustering. It is suggested in the pioneering works of Refs. [4,5] that the nonuniform distributions are caused by preferential concentration where the particles are centrifuged out from vortical flows and accumulated in straining flows. A typical quantity characterizing the particle clustering is the two-point correlation of particle number density, called a pair-correlation function (PCF). Since PCF is a function of the separation distance $r$ of the two points, it quantifies the clustering at each scale $r$ and we can analyze the scale dependence of the clustering through PCF.

Let us recall that eddies with variable sizes ranging from an integral length scale $L$ to the Kolmogorov scale $\eta$ reside in turbulence and show strong nonlinear interaction. Here $L$ and $\eta$ respectively characterize the typical length scales of the energy-containing and dissipation ranges. In an intermediate scale range between $L$ and $\eta$, called the inertial range, conservative quantities, e.g., energy, cascades to smaller scales due to dominant scale-local transfer. According to the idea by Kolmogorov in 1941 [6] (hereafter referred to as K41), turbulence may have a certain universality in the inertial range at sufficiently high Reynolds numbers in the sense that statistics, e.g., scaling exponents and proportional constants in correlation functions, are invariant and free from the properties of both energy-containing and dissipation ranges.

It is well expected that the universality of turbulence statistics is reflected in particle clustering, especially in the scaling of PCF. Indeed, a number of studies have been devoted so far to particle-laden turbulence with its relation to K41. Elperin et al. [7] and Balkovsky et al. [8] analyzed particles advected by modeled velocity fields subjected to given power spectra and short correlation time. Especially in Ref. [8], a certain dependence of PCF on $r$ is suggested, while Bragg et al. [9] obtained a similar result using a different closure model [10]. There are some studies from the structural point of view; particle's voids [11] and fluid's zero-acceleration points swept by turbulence [12] were investigated with there relations to scale-dependent clustering in the inertial range. Fourier and wavelet analyses $[13,14]$ were also conducted to extract scale dependence and structures of the number-density field. In addition to theoretical and numerical studies, experiments are also performed; Ref. [15] detected a broad scaling range of clustering in the inertial range while scale-similar clustering in the dissipation range was also confirmed. These works support the multiscale aspect of particle clustering ranging over the inertial range. However, unlike the well-known universality in turbulence statistics, an explicit scale similarity of particle clustering in the inertial range has not been clarified yet.

In this paper, we find an explicit power law of PCF in the inertial range by using a perturbation analysis and a closure theory. The structure of this paper is as follows. The basic equations are given in Sec. II. In Sec. III, we derive the universal scaling law for PCF in the inertial range through perturbation and dimensional analysis. In Sec. IV, the same scaling law is obtained with the estimate of the universal constant using a closure theory. The dynamical interpretation of the scaling law is also given. In Sec. V, the derived power law of PCF is verified through a direct numerical simulation (DNS). Conclusions and discussions are given in Sec. VI.

\section{EQUATIONS OF PARTICLE NUMBER-DENSITY FIELD}

In this study, we consider heavy particles subjected only to the Stokes force $[4,16]$ and neglect their counteraction to fluid and interactions between particles. By using the spatial filtering of a length scale $\ell$, we obtain the coarse-grained

\footnotetext{
*tariki@nagoya-u.jp

†yoshida.kyo.fu@u.tsukuba.ac.jp

${ }^{\ddagger}$ k.matsuda@jamstec.go.jp

§yosimatu@imass.nagoya-u.ac.jp
} 
number-density $n(\mathbf{x}, t)$ and particle velocity $\mathbf{v}(\mathbf{x}, t)$; these are governed by the following equations:

$$
\begin{aligned}
\partial_{t} n+\partial_{j}\left(n v_{j}\right) & =-\partial_{j} q_{j}, \\
\left(\partial_{t}+v_{j} \partial_{j}\right) v_{i} & =-\left(v_{i}-u_{i}\right) / \tau_{p}-\partial_{j} \sigma_{i j},
\end{aligned}
$$

where $\partial_{t} \equiv \partial / \partial_{t}, \partial_{j} \equiv \partial / \partial x_{j}, \tau_{p}$ is the relaxation time scale of relative velocity between particles and fluid and $\mathbf{u}$ is the fluid velocity governed by the Navier-Stokes equation and the incompressibility condition:

$$
\begin{aligned}
\left(\partial_{t}+u_{j} \partial_{j}\right) u_{i} & =-\partial_{i} P+v \nabla^{2} u_{i}, \\
\partial_{j} u_{j} & =0,
\end{aligned}
$$

where $P$ is the pressure and $v$ is the kinematic viscosity. Note that $q_{i}$ and $\sigma_{i j}$ express effective fluxes caused by subfilter clustering, which may be not significant at sufficiently larger scales $(\gg \ell)$. In the following discussions, we always choose $\ell$ to be sufficiently smaller than the scale of our interest, where $q_{i}$ and $\sigma_{i j}$ are consistently neglected.

\section{PERTURBATION ANALYSIS}

Here we introduce the normalized number-density fluctuation $\theta(\equiv(n-\langle n\rangle) /\langle n\rangle)$ and formally expand $\mathbf{v}$ and $\theta$ in terms of $\tau_{p}$ :

$$
\theta=\sum_{N=0}^{\infty} \tau_{p}^{N} \theta^{(N)}, \quad v_{i}=\sum_{N=0}^{\infty} \tau_{p}^{N} v_{i}^{(N)},
$$

where $v_{i}^{(0)}=u_{i}$. To extract the nontrivial distribution due to particle's nonzero inertia $\left(\tau_{p} \neq 0\right)$, we initially set $\theta^{(0)}=0$ so that it will remain zero since $\left(\partial_{t}+u_{j} \partial_{j}\right) \theta^{(0)}=0$. Substitution of Eq. (3) into Eqs. (1a) and (1b) yields a dynamical equation for $\theta^{(N)}$ of each order $N(\in \mathbb{N})$. In particular,

$$
\left(\partial_{t}+u_{j} \partial_{j}\right) \theta^{(1)}=\partial_{j} u_{i} \partial_{i} u_{j}
$$

expresses the preferential concentration [4,5], where Eq. (2b) is used. Here we introduce $\mathrm{PCF} \Theta(\mathbf{r})(\equiv\langle\theta(\mathbf{x}+\mathbf{r}) \theta(\mathbf{x})\rangle)$ as an indicator for clustering at scale $r$ [1]. PCF can be expanded in terms of $\tau_{p}$ :

$$
\Theta(\mathbf{r})=\tau_{p}^{2}\left\langle\theta^{(1)}(\mathbf{x}+\mathbf{r}) \theta^{(1)}(\mathbf{x})\right\rangle+O\left(\tau_{p}^{3}\right) .
$$

Next, we focus on the inertial range of fully developed isotropic turbulence, that is, $\eta \ll r \ll L$, where $r(\equiv|\mathbf{r}|)$ is the distance between two points, $L$ is the energy-containing scale, $\eta$ (三 $\left.v^{3 / 4} \epsilon^{-1 / 4}\right)$ is the Kolmogorov scale, and $\epsilon$ is the mean energy dissipation rate. Since every $\theta^{(N)}$ is related to the fluid velocity $\mathbf{u}$, we expect their moments to be scaled by $\epsilon$ and $r$; this is referred to as the K41 scaling hereafter [6]. Equation (5) is thus rewritten as $\Theta(r)=\sum_{N=2} C_{N} S_{r}^{N}$, where $C_{N}$ is the universal constant with the assumption of K41 scaling; $S_{r} \equiv \tau_{p} / \tau_{r}=$ $\tau_{p} \epsilon^{1 / 3} r^{-2 / 3}$ is the scale-dependent Stokes number [17,18]; and $\tau_{r}\left(\equiv \epsilon^{-1 / 3} r^{2 / 3}\right)$ is the turbulence time scale. Now, the formal expansion in $\tau_{p}$ can be interpreted as the expansion in $S_{r}$. In the case of $r \gg \Lambda\left(\equiv \tau_{p}^{3 / 2} \epsilon^{1 / 2}\right), S_{r}$ is sufficiently smaller than unity, so we obtain the scale-similar law as follows:

$$
\Theta(r)=C_{2} \tau_{p}^{2} \epsilon^{2 / 3} r^{-4 / 3}(\max [\eta, \Lambda] \ll r \ll L) .
$$

In the above-mentioned discussion, the filter scale $\ell$ is presupposed to satisfy $\ell \ll \max [\eta, \Lambda]$ so that subfilter properties do not affect the physics of our interest.

Balkovsky et al. [8] and Bragg et al.[9] studied the particle clustering in terms of the radial-distribution function (RDF) $R(r)$ which can be related to PCF as $R(r)=1+\Theta(r)$. (Note that RDF in this paper is called PCF in Ref. [8].) Relying on delta-correlated Gaussian velocity, Ref. [8] suggested $\log R(r) \propto a^{4} \eta^{-8 / 3} r^{-4 / 3}$ (a: particle radius) under limited Stokes number $S_{\eta} \ll 1$. On the other hand, using Gaussian assumption of the velocity increment [10], Ref. [9] proposed $R(r)=\exp \left(\mathcal{D} r^{-4 / 3}\right)$ with $\mathcal{D}$ being an unknown function of $S_{\eta}$. Up to the leading-order of an expansion $\Theta(r)=R(r)-$ $1=A r^{-4 / 3}+O\left(r^{-8 / 3}\right)\left(A=\right.$ const. $\left.\times a^{4} \eta^{-8 / 3}, \mathcal{D}\right)$, their results are consistent with Eq. (6). Our analysis in this section implies that the leading-order behavior of PCF or RDF for large $r$ can be derived from a simple perturbation and dimensional analysis. The present perturbation analysis may be extended to higher-order terms; however, the resulting coefficients can be, in general, different from the exponential form obtained in Refs. [8,9]. Since the perturbation analysis is valid only for large $r$, the leading-order term and its coefficient $C_{2}$ is of the highest interest. We will discuss a theoretical analysis to evaluate $C_{2}$ in Sec. IV.

\section{CLOSURE THEORY OF PCF}

\section{A. Lagrangian-renormalized approximation}

The scale-similar law (6), while being uniquely determined through the dimensional analysis, can also be understood from the dynamical aspect with the help of so-called two-point closure approximations. Furthermore, the two-point closures are capable of quantitatively estimating universal constants, such as $C_{2}$, in Eq. (6). Among various closure theories, we choose the Lagrangian renormalized approximation (LRA) [19], which is known for its remarkable successes in the homogeneous isotropic turbulence, feasibly deriving the Kolmogorov spectrum with its universal constant [20].

By neglecting the higher-order term $\theta^{(N)}(N \geqslant 2)$ in Eqs. (3) and (4), we obtain

$$
\left(\partial_{t}+u_{j} \partial_{j}\right) \theta=\tau_{p} \partial_{i} u_{j} \partial_{j} u_{i}=-\tau_{p} \nabla^{2} P,
$$

where we used Eq. (2a). Now $\theta$ is expressed as a passive scalar subjected to a pressure-dependent source term [21-23], in which the pressure statistics play a key role, as discussed in the later discussions. Next, we apply LRA method to Eq. (7). In the standard formalism, LRA introduces the Lagrangian variable of $\theta$, that is, $\theta\left(\mathbf{x}, t^{\prime} \mid t\right)$, which is the value of $\theta$ experienced at time $t$ by a fluid element located on $\mathbf{x}$ at time $t^{\prime}$. The closure equations are then obtained in terms of the Lagrangian correlation function $\Theta\left(\mathbf{r} ; t, t^{\prime}\right)=\left\langle\theta\left(\mathbf{x}+\mathbf{r}, t^{\prime} \mid t\right) \theta\left(\mathbf{x}, t^{\prime}\right)\right\rangle$. However, LRA based on $\theta$ often overestimates the turbulence mixing because of the lack of the memory-fading effect in the two-time correlation $\Theta\left(\mathbf{r} ; t, t^{\prime}\right)[20,24]$, i.e., the correlation time scale $\int_{t^{\prime}}^{\infty} d t \Theta\left(\mathbf{r} ; t, t^{\prime}\right) / \Theta\left(\mathbf{r} ; t^{\prime}, t^{\prime}\right)$ far exceeds the turbulence time scale $\tau_{r}=\epsilon^{-1 / 3} r^{2 / 3}$. This is solely because the scalar itself does not change its value under the strain of fluid, suggesting that we need to choose a dynamical variable, other than $\theta$, representing the memory-fading effect. One of possible 
candidates is a nonlocal expression of Hessian $\partial_{i} \partial_{j} \theta$ spatially integrated by the Laplacian inverse $\nabla^{-2}$, that is, $\nabla^{-2} \partial_{i} \partial_{j} \theta$. This expression reflects the memory fading under the straining motion of a fluid. Since the nonlocal Hessian contains $\theta$ in its trace part, the remaining traceless part, that is,

$$
\mathscr{H}_{i j} \equiv\left(\nabla^{-2} \partial_{i} \partial_{j}-\frac{1}{3} \delta_{i j}\right) \theta
$$

may be a desired variable consistent with the memory fading effect (see Appendix for more detailed discussions). By applying LRA to $\mathscr{H}_{i j}$, we obtain another closure model based on the Lagrangian correlation $\mathscr{H}_{i j l m}\left(\mathbf{r} ; t, t^{\prime}\right) \equiv$ $\left\langle\mathscr{H}_{i j}\left(\mathbf{x}+\mathbf{r}, t^{\prime} \mid t\right) \mathscr{H}_{l m}\left(\mathbf{x}, t^{\prime}\right)\right\rangle$ and the Lagrangian response function $\mathscr{G}_{i j l m}\left(\mathbf{r} ; t, t^{\prime}\right)$ of $\mathscr{H}_{i j}$. Let us introduce the contraction $\mathscr{H}\left(\mathbf{r} ; t, t^{\prime}\right) \equiv 3 \mathscr{H}_{i j i j}\left(\mathbf{r} ; t, t^{\prime}\right) / 2$. The important property of $\mathscr{H}\left(\mathbf{r} ; t, t^{\prime}\right)$ is that the equal-time correlation is identical to PCF, i.e., $\mathscr{H}(\mathbf{r} ; t, t)=\Theta(\mathbf{r}, t)=\Theta(\mathbf{r} ; t, t)$. However, note that $\mathscr{H}\left(\mathbf{r} ; t, t^{\prime}\right) \neq \Theta\left(\mathbf{r} ; t, t^{\prime}\right)$ for $t \neq t^{\prime}$, in general. We refer to this closure strategy based on Hessian as $H$-based LRA in contrast to the conventional $\theta$-based LRA. By following the procedures outlined in Appendix, the $H$-based LRA yields a closed set of integrodifferential equations containing $H_{i j l m}\left(\mathbf{k} ; t, t^{\prime}\right)$ $\left(\equiv \mathcal{F}_{\mathbf{r}}^{\mathbf{k}} \mathscr{H}_{i j l m}\left(\mathbf{r} ; t, t^{\prime}\right)\right), G_{i j l m}\left(\mathbf{k} ; t, t^{\prime}\right)\left(\equiv \mathcal{F}_{\mathbf{r}}^{\mathbf{k}} \mathscr{G}_{i j l m}\left(\mathbf{r} ; t, t^{\prime}\right)\right), \quad$ and $Q_{i j}\left(\mathbf{k} ; t, t^{\prime}\right)\left[\equiv \mathcal{F}^{\mathbf{k}}\left\langle u_{i}\left(\mathbf{x}+\mathbf{r}, t^{\prime} \mid t\right) u_{j}\left(\mathbf{x}, t^{\prime}\right)\right\rangle\right]$, where $\mathcal{F}_{\mathbf{r}}^{\mathbf{k}} f(\mathbf{r})[\equiv$ $\left.(2 \pi)^{-3} \int d \mathbf{r} \mathrm{e}^{-i \mathbf{k} \cdot \mathbf{r}} f(\mathbf{r})\right]$ represents the Fourier transformation of an arbitrary physical-space function $f(\mathbf{r})$. For isotropic cases, the closure equations are written in terms of the spectral functions $H\left(k ; t, t^{\prime}\right) \equiv(3 / 2) H_{i j i j}\left(\mathbf{k} ; t, t^{\prime}\right)$, $G_{H}\left(k ; t, t^{\prime}\right) \equiv G_{i j i j}\left(\mathbf{k} ; t, t^{\prime}\right) \quad$ and $\quad Q\left(k ; t, t^{\prime}\right) \equiv(1 / 2)\left(\delta_{i j}-\right.$ $\left.k_{i} k_{j} / k^{2}\right) Q_{i j}\left(\mathbf{k} ; t, t^{\prime}\right)$ with $k \equiv|\mathbf{k}|$. The resultant equation for $H(k ; t, t)$ is given by

$$
\partial_{t} H(k ; t, t)=T(k, t)+\Psi(k, t),
$$

where

$$
\begin{aligned}
T(k, t)= & 2 \pi \iint_{I_{k}} d p d q k p q\left(1-y^{2}\right) \int_{-\infty}^{t} d s Q(q ; t, s) \\
& \times\left\{H(p ; t, s) G_{H}(k ; t, s)-H(k ; t, s) G_{H}(p ; t, s)\right\},
\end{aligned}
$$

$$
\Psi(k, t)=\tau_{p}^{2} \frac{k^{2}}{4 \pi} \int_{-\infty}^{t} d s G_{H}(k ; t, s) E_{p}(k ; t, s),
$$

where, for wave-number integration, we defined the following geometrical properties: $y \equiv\left(q^{2}+\right.$ $\left.k^{2}-p^{2}\right) /(2 k q), z \equiv\left(p^{2}+k^{2}-q^{2}\right) /(2 k p), \quad$ and $\quad I_{k} \equiv$ $\{(p, q)|| k-p \mid \leqslant q \leqslant k+p\}$. Here $T(k, t)$ expresses the transfer of scalar-variance due to turbulence mixing. The turbulence mixing is essentially caused by the velocity statistics $Q(q ; t, s)$. However, note that the scalar statistics $H(p ; t, s)$ at various wave number $p$ also contribute to the transfer $T(k, t)$ of the scalar variance at wave number $k . \Psi(k, t)$ acts as the source term driving the preferential concentration. A spectral function $E_{p}(k ; t, s)$ is given by

$$
\begin{aligned}
E_{p}(k ; t, s) \equiv & 8 \alpha \pi^{2} \iint_{I_{k}} d p d q k p q\left(1-y^{2}\right) \\
& \times\left(1-z^{2}\right) Q(p ; t, s) Q(q ; t, s),
\end{aligned}
$$

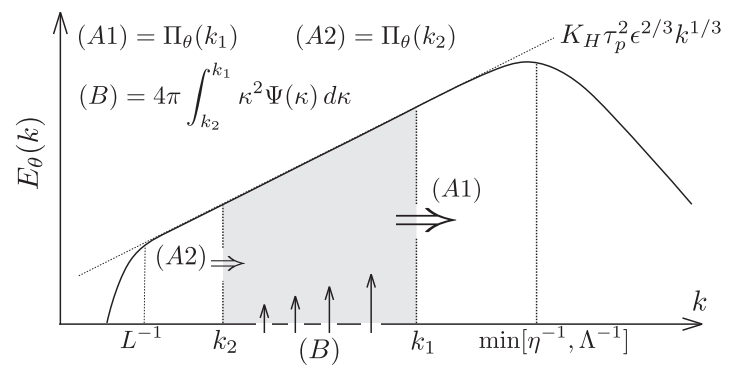

FIG. 1. Balance between turbulence mixing and preferential concentration is schematically shown in $k$ space. The budget of the number-density variance in the wave-number band $\left[k_{2}, k_{1}\right]$ (gray area) reads $-(A 1)+(A 2)+(B)=0$, expressing the balance between total flux $-(A 1)+(A 2)$ and source $(B)$ due to preferential concentration.

which is related to the pressure spectrum by $E_{p}(k, t)=$ $E_{p}(k ; t, t)$, where $\left\langle P^{2}\right\rangle=\int_{0}^{\infty} E_{p}(k, t) d k$ and the parameter $\alpha=1$ is introduced for a later discussion. The remaining equations for $H\left(k ; t, t^{\prime}\right)$ and $G_{H}\left(k ; t, t^{\prime}\right)$ are given by Eqs. (A9) and (A10) in Appendix.

Since we focus on the inertial range, we apply the scalesimilar form $Q\left(k ; t, t^{\prime}\right)=\left(K_{o} / 2 \pi\right) \epsilon^{2 / 3} k^{-11 / 3} g\left(\epsilon^{1 / 3} k^{2 / 3} \mid t-\right.$ $\left.t^{\prime} \mid\right)$ to the closure equations, where $K_{o} \approx 1.72$ and $g(\tau)$ is the dimensionless function identical to $R(x)$ of Eq. (27) in Ref. [20]. Then, the scale-similar solutions

$$
\begin{array}{r}
H\left(k ; t, t^{\prime}\right)=\frac{K_{H}}{4 \pi} \tau_{p}^{2} \epsilon^{2 / 3} k^{-5 / 3} f_{H}\left(\epsilon^{1 / 3} k^{2 / 3}\left(t-t^{\prime}\right)\right), \\
G_{H}\left(k ; t, t^{\prime}\right)=g_{H}\left(\epsilon^{1 / 3} k^{2 / 3}\left(t-t^{\prime}\right)\right)\left(t \geqslant t^{\prime}\right),
\end{array}
$$

are consistent with the closure equations, where $f_{H}(\tau)$ and $g_{H}(\tau)$ are dimensionless functions satisfying $f_{H}(0)=$ $g_{H}(0)=1$. The number-density spectrum is given by $E_{\theta}(k)=$ $4 \pi k^{2} H(k ; t, t)$, whose scale-similar solution is written as $E_{\theta}(k)=K_{H} \tau_{p}^{2} \epsilon^{2 / 3} k^{1 / 3}$ by using another universal constant $K_{H}$. The functions $f_{H}(\tau), g_{H}(\tau)$ and the constant $K_{H}$ can be numerically solved, satisfying $f_{H}(\tau), g_{H}(\tau) \rightarrow 0(\tau \rightarrow$ $\infty)$. This implies that $H$-based LRA properly incorporates the memory-fading effect relevant to turbulence mixing. In the physical space, PCF is related to $H(k ; t, t)$ as $\Theta(r, t)=$ $4 \pi \int_{0}^{\infty} k r^{-1} \sin (k r) H(k ; t, t) d k$ so the universal constant $C_{2}$ of Eq. (6) is given by $C_{2}=\Gamma(1 / 3) K_{H} / 2$; hence,

$$
K_{H} \approx 6.7, \quad C_{2} \approx 9.0 .
$$

Note that conventional $\theta$-based LRA yields substantially smaller value $C_{2} \approx 2.6$.

\section{B. Interscale dynamics}

To obtain deeper insights of the scale-similar clustering, we discuss the interscale dynamics of the number-density spectrum $E_{\theta}(k)$ according to Eq. (9). Under the statistically stationary condition, we consider the budget of the numberdensity variance in a wave-number band $\left[k_{2}, k_{1}\right]$ through the spherical integration of Eq. (9); $0=-\Pi_{\theta}\left(k_{1}\right)+\Pi_{\theta}\left(k_{2}\right)+$ $4 \pi \int_{k_{2}}^{k_{1}} \kappa^{2} \Psi(\kappa) d \kappa$, as depicted in Fig. 1. Here $\Pi_{\theta}(k) \equiv$ $-4 \pi \int_{0}^{k} \kappa^{2} T(\kappa) d \kappa$ defines the flux of the number-density variance from the lower to higher wave-number regions separated 
by $k$. In the scale-similar range $L^{-1} \ll k \ll \min \left[\eta^{-1}, \Lambda^{-1}\right]$, we obtain the forward flux $\Pi_{\theta}(k)=C_{\Pi} \tau_{p}^{2} \epsilon k^{2}>0\left(C_{\Pi} \approx 1.8\right)$, where the number-density variance excited by the preferential concentration are carried to the smaller scale through turbulence mixing, causing the dynamical balance of the numberdensity statistics. In physical space, clusters caused by the preferential concentration at various scales are broken into smaller pieces because of the turbulence mixing.

\section{NUMERICAL SIMULATION}

To discuss the reliability of our prediction, we calculated the PCF by using turbulent clustering data obtained through a three-dimensional DNS of particle-laden isotropic turbulence, in which discrete particles were tracked using the Lagrangian method. (See Refs. [13,25] for details of the computational method.) The number of grid points for the flow field was set to $N_{g}^{3}=1000^{3}$ and the Reynolds number based on the representative length and velocity scales was set to $\operatorname{Re}(\equiv$ $\left.L_{0} U_{0} / v\right)=2220$. The RMS value of velocity fluctuation $u^{\prime}$ and the Taylor-microscale-based Reynolds number $\operatorname{Re}_{\lambda}$ (三 $u^{\prime} \lambda / \nu$, where $\lambda$ is the Taylor microscale), were $u^{\prime} / U_{0}=0.992$ and $\operatorname{Re}_{\lambda}=328$, respectively. The grid-point number $N_{g}^{3}$ was sufficiently large for resolving the turbulent flow so that $k_{\max } \eta$ reached $2.08\left(k_{\max } \equiv N_{g} / 2 L_{0}\right)$. The number of particles was set to $5 \times 10^{7}$ and the Stokes number was set to $S_{\eta}=1.0$, implying $\Lambda=\eta$.

The PCF in Fig. 2 is obtained by averaging 10 independent PCFs at 10 time points equally spaced in a time interval longer than turbulence eddy-turnover time. The standard deviation of PCF is atmost $3 \%$ in $10 \lesssim r / \eta \lesssim 100$. According to Fig. 2, PCF approximately obeys the scaling $\Theta(r) \propto r^{-4 / 3}$ of Eq. (6) in $10 \lesssim r / \eta \lesssim 200$. In our DNS, the velocity statistics obeys K41 scaling for $75 \lesssim r / \eta \lesssim 200$, which may be regarded as the inertial range, while the region $10 \lesssim r / \eta \lesssim 75$ may be contaminated by the bottleneck effect [26,27]. Namely, -4/3power behavior of PCF extends to the scale of the bottleneck effect. The proportional constant is estimated through $C_{2}^{\mathrm{DNS}}=$

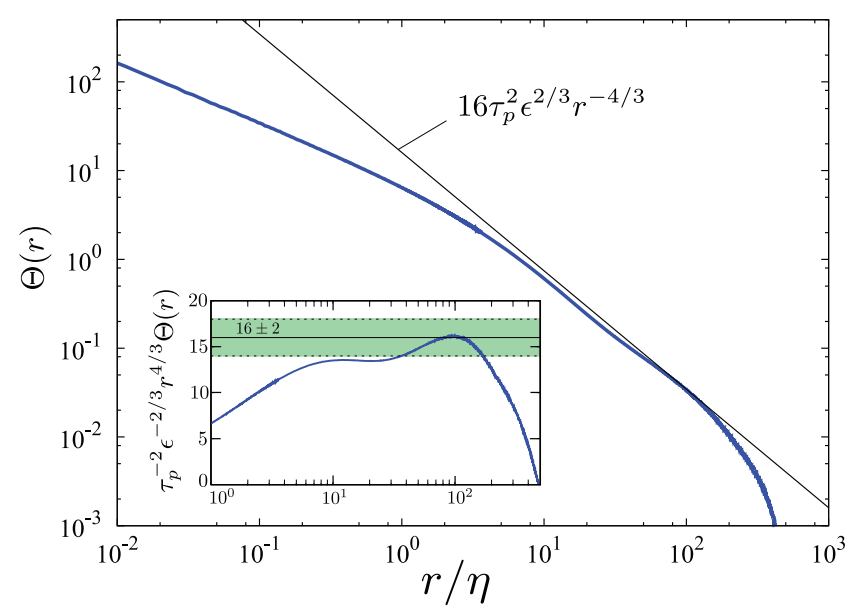

FIG. 2. Pair correlation function (PCF) from the DNS. The inset shows the compensated PCF $\left[\tau_{p}^{-2} \epsilon^{-2 / 3} r^{4 / 3} \Theta(r)\right]$ which gently varies from 13 to 16 for $75 \lesssim r / \eta \lesssim 200$, being comparable with theoretical estimation $16 \pm 2$ of Eq. (16) (green band). $\tau_{p}^{-2} \epsilon^{-2 / 3} r^{4 / 3} \Theta(r)$, which gently varies from 13 to 16 for $75 \lesssim r / \eta \lesssim 200$ (inset of Fig. 2 ). Compared with $C_{2} \approx 2.6$ of $\theta$-based LRA, $C_{2} \approx 9.0$ of the $H$-based LRA is closer to the DNS result. However, a discrepancy of a 1.5 factor still exists between DNS and $H$-based LRA results. Here, note that the preferential concentration is firmly related to the pressure correlation in Eq. (11). Our DNS suggests $K_{p}^{\text {DNS }} \approx 6.9 \pm 0.9$ for the pressure spectrum $E_{p}(k)=K_{p} \epsilon^{4 / 3} k^{-7 / 3}$, while $H$ based LRA gives $K_{p}^{\mathrm{LRA}} \approx 3.9$. A similar trend is reported in Ref. [28] $\left(K_{p}^{\mathrm{DNS}}=8.0 \pm 0.5\right.$ for $\left.\operatorname{Re}_{\lambda}=387,460,478\right)$, where the pressure spectrum, which appreciably reflects the nonGaussianity, is underestimated by Gaussian-based analyses such as $H$-based LRA. This would also cause the underestimation of $\Psi(k)$ based on $E_{p}\left(k ; t, t^{\prime}\right)$ in Eq. (11). Here we simply restore $\Psi(k)$ by setting $\alpha=K_{p}^{\mathrm{DNS}} / K_{p}^{\mathrm{LRA}}=1.8 \pm 0.2$ in Eq. (12). Then, instead of Eq. (15), $H$-based LRA yields

$$
C_{2} \approx 9.0 \alpha \approx 16 \pm 2
$$

which reasonably estimates the DNS result.

For further progress of the current analysis, there are two possibilities: (i) to perform DNS of higher Reynolds numbers and to determine whether the observed $C_{2}$ and $K_{p}$ approach the LRA values or (ii) to incorporate non-Gaussianity into $E_{p}(k)$ and $\Psi(k)$ of the theory. Regarding (i), we should remark the necessity of much higher Reynolds numbers for the pressure statistics. As remarked in Ref. [28], the pressure spectrum just begins to obey $\mathrm{K} 41$ scaling from $\mathrm{Re}_{\lambda} \gtrsim 300$, while it becomes clearer for $\operatorname{Re}_{\lambda} \gtrsim 600$ [29]. Thus, we can expect to observe a clearer $-4 / 3$ power law in PCF for $\operatorname{Re}_{\lambda} \gtrsim 600$. Furthermore, the coefficient $C_{2}$ may reach the universal constant when $K_{p}$ saturates at sufficiently high Reynolds numbers; this has not been confirmed yet through both simulations and experiments [29]. Thus, the true verification of the universal $C_{2}$ will be left as an open question until we reach even higher Reynolds numbers, while Eq. (16) serves as a tentative relation between observed $C_{2}$ and $K_{p}$ for moderate Reynolds numbers. For further detailed analysis for moderate Reynolds numbers, one may utilize DNS data of $E_{p}\left(k ; t, t^{\prime}\right)$ instead of assuming its scale-similar form, which could evaluate at each scale the deviation of observed PCF from the theoretical result (6).

\section{CONCLUSIONS AND DISCUSSIONS}

We have investigated the large-scale behavior of particle clustering and obtained the $-4 / 3$ power law Eq. (6) of PCF based on a dimensional analysis and a Lagrangian closure, LRA. The LRA further elucidated the forward flux of the number-density variance competing with the preferential concentration. The scale-similar law of PCF is also supported by our DNS of particle-laden isotropic turbulence, in which the obtained PCF reaches close to the $-4 / 3$ power curve. Theoretical analysis based on LRA reasonably predicts the constant of the power law, where the theory gives $C_{2}=16 \pm 2$ while the DNS suggests $C_{2}=13-16$. This quantitative success is mostly due to the present $H$-based LRA, an alternative closure to the conventional $\theta$-based LRA for passive-scalar turbulence. The $H$-based LRA properly evaluates the turbulence-mixing effect and it would contribute to more general problems associated with passive scalar turbulence, e.g., quantitative 
analysis of the scalar spectrum in the inertial-convective range, the inertial-diffusive range, and the viscous-convective range. Also $H$-based LRA has an extendability in particle clustering subjected to general turbulence where $Q\left(k ; t, t^{\prime}\right)$ and $E_{p}\left(k ; t, t^{\prime}\right)$ in Eqs. (9)-(11) deviate from inertial-range spectra, which would predict the deviation from the power law $\Theta(r)=C_{2} \tau_{p}^{2} \epsilon^{3 / 2} r^{-4 / 3}$. Such generalization may be useful in atmospheric or oceanic sciences, where density stratification, gravitation, or system rotation may occur.

In the present study, we have focused on the net budget, or the mean flux, of the number-density variance in the wave-number space in Sec. IV B. The qualitative achievements obtained for PCF and number-density spectrum in the present study suggest that the analysis of the mean flux is sufficient for the evaluations of the second-order moments such as the PCF and the number-density spectrum. For the analysis for the higher-order moments and the intermittent structure of the particle clustering at small scales, it would be important to take into account of the fluctuation of the flux. A possible future study would be to investigate the intermittency in the particle clustering through a multiplicative stochastic process model of the number-density flux in analogy with the analysis of the intermittency of velocity statistics that originates from Kolmogorov's study in 1962 [30].

Finally, we discuss the significance of the positive-exponent power law of the number-density spectrum $E_{\theta}(k) \propto k^{1 / 3}$ which is divergent at high wave number, i.e., at small scale. Since the present analysis is based on perturbation for small $S_{r}$, it is expected that the divergent behavior of $E_{\theta}(k)$ is restricted in the range $k<\Lambda^{-1}$. This consideration suggests an extendability of the present analysis to give important insights in the collision physics at small scales. Recall that $\Lambda$ is the characteristic length at which the relaxation time scales of particle $\tau_{p}$ and turbulence $\tau_{r}$ are of the same order. Note the qualitative change in the clustering around this scale; inertial particles mostly move along with the large eddies of $r>\Lambda\left(\tau_{p}<\tau_{r}\right)$, while they may be relatively insensitive to the small eddies of $r<\Lambda\left(\tau_{p}>\tau_{r}\right)$. This may be reflected in the number-density spectrum $E_{\theta}(k)$, the behavior of which may change at the scale of $k \sim \Lambda^{-1}$. It is suggested by a DNS [13] that $E_{\theta}(k)$ has a peak around $k \sim \Lambda^{-1}$ and decrease with the increase of $k$ in the range $k>\Lambda^{-1}$ as depicted in Fig. 1. We should remark that RDF at $r=d_{p}$ (particle's diameter), that is, $R\left(d_{p}\right)=1+\Theta\left(d_{p}\right) \approx$ $\Theta\left(d_{p}\right)=\int_{0}^{\infty} E_{\theta}(k) \sin \left(k d_{p}\right) /\left(k d_{p}\right) d k$, is an essential factor for the collision rate [31,32]. If $E_{\theta}(k)$ rapidly decreases for $k \gg \Lambda^{-1}, R\left(d_{p}\right)$ is mostly determined by the properties of $k \lesssim \Lambda^{-1}$ where the $-4 / 3$ power law holds. This approach should be further pursued in future works.

\section{ACKNOWLEDGMENTS}

T.A. is sincerely thankful to Prof. T. Gotoh for his valuable comments on the LRA formalism and its application to particle clustering. K. Yoshida is grateful to Prof. M. Umemura for useful discussions and comments on particle clustering in accretion disks. This work was supported by JSPS Grantin-Aid for Scientific Research (S) 16H06339. The numerical simulation presented here was conducted on the Earth Simulator supercomputer system operated by the Japan Agency for Marine-Earth Science and Technology.

\section{APPENDIX: H-BASED LRA}

The Lagrangian correlation of the particle-number density, i.e., $\Theta\left(r ; t, t^{\prime}\right)$, does not reflect the memory-fading effect caused by turbulence mixing. This is because $\theta$ in the Lagrangian picture is insensitive to random scrambling of fluid elements. In order to incoorporate the memory-fading effect, we need to choose an alternative field variable representing memory fading reflecting the scrambling motion.

One may choose the spatial derivatives of $\theta$ as such possible choices. In regard to the scalar gradient $\partial_{i} \theta$, the simplest derivative, Ref. [24] had pointed out that its Lagrangian correlation does not show sufficient memory fading, implying that the variable is not appropriate for our aim. Then we focus on the Hessian $\partial_{i} \partial_{j} \theta$ as the second simplest choice. Reminding that the derivative operations often emphasize small-scale structures of sub-Kolmogorov scale, an integrated variable $\nabla^{-2} \partial_{i} \partial_{j} \theta\left(=\mathscr{H}_{i j}^{\text {tot }}\right)$ may well be chosen. It should be noted, however, that $\mathscr{H}_{i j}^{\text {tot }}$ contains $\theta$ in its trace part (i.e., $\mathscr{H}_{i i}^{\text {tot }}=\theta$ ), which may suffer from long-time memory as well as $\theta$ does. Thus, we expect that the traceless part of the nonlocal Hessian $\mathscr{H}_{i j}^{\text {tot }}-\frac{1}{3} \theta \delta_{i j}\left(\equiv \mathscr{H}_{i j}\right)$ is appropriate variable of our closure analysis.

Next, we consider the dynamical equation of $\mathscr{H}_{i j}$, which is obtained from Eq. (7):

$$
\partial_{t} \mathscr{H}_{i j}=\lambda\left(\nabla^{-2} \partial_{i} \partial_{j}-\frac{1}{3} \delta_{i j}\right)\left(-u_{l} \partial_{l} \theta+\tau_{p} \partial_{l} u_{m} \partial_{m} u_{l}\right),
$$

where $\lambda=1$ is a bookkeeping parameter. In the same manner, we apply $\lambda$ to all the nonlinear terms in dynamical equations of the system regarding nonlinear terms as perturbations. We choose the Lagrangian correlations $\quad$ [i.e., $\quad Q_{i j}\left(\mathbf{r} ; t, t^{\prime}\right) \equiv\left\langle u_{i}\left(\mathbf{x}+\mathbf{r}, t^{\prime} \mid t\right) u_{j}\left(\mathbf{x}, t^{\prime}\right)\right\rangle$ and $\left.\quad \mathscr{H}_{i j l m}\left(\mathbf{r} ; t, t^{\prime}\right) \equiv\left\langle\mathscr{H}_{i j}\left(\mathbf{x}+\mathbf{r}, t^{\prime} \mid t\right) \mathscr{H}_{l m}\left(\mathbf{x}, t^{\prime}\right)\right\rangle\right] \quad$ and correspinding Lagrangian-response functions [i.e., $G_{i j}\left(\mathbf{r} ; t, t^{\prime}\right)$ and $\left.\mathscr{G}_{i j l m}\left(\mathbf{r} ; t, t^{\prime}\right)\right]$ as the representative variables to close the system of equations, where these representative may be written in abbreviated forms $Q, \mathscr{H}, G$, and $\mathscr{G}$ in this order. Once the representatives are chosen, an arbitrary unclosed correlation is expressed by the representative variables following the LRA procedure [19] outlined as follows.

(i) Assume the linearlized (nonperturbative) solution of $u_{i}$ and $\mathscr{H}_{i j}$ to be Gaussian random. Then an arbitrary correlation $J$ of the system can be expanded in terms of the second-order correlations $(\tilde{Q}$ and $\tilde{\mathscr{H}})$ and the response functions $(\tilde{G}$ and $\tilde{\mathscr{G}})$ of the linearlized fields:

$$
J=\mathcal{J}^{(0)}[\tilde{Q}, \tilde{G}, \tilde{\mathscr{H}}, \tilde{\mathscr{G}}]+\lambda \mathcal{J}^{(1)}[\tilde{Q}, \tilde{G}, \tilde{\mathscr{H}}, \tilde{\mathscr{G}}]+O\left(\lambda^{2}\right)
$$

where $\mathcal{J}^{(n)}(n=0,1,2, \ldots)$ are functionals. Here, we use the relation $\tilde{\Theta}=(3 / 2) \tilde{\mathscr{H}}_{i j i j}$ to eliminate the linearized solution of PCF $\tilde{\Theta}$.

(ii) Similarly, expand $Q, G, \mathscr{H}$, and $\mathscr{G}$ in terms of $\tilde{Q}, \tilde{G}, \tilde{\mathscr{H}}$, and $\tilde{\mathscr{G}}$ :

$$
\begin{aligned}
Q & =\tilde{Q}+\lambda^{2} \mathcal{A}^{(2)}[\tilde{Q}, \tilde{G}]+O\left(\lambda^{4}\right), \\
G & =\tilde{G}+\lambda^{2} \mathcal{B}^{(2)}[\tilde{Q}, \tilde{G}]+O\left(\lambda^{4}\right), \\
\mathscr{H} & =\tilde{\mathscr{H}}+\lambda^{2} \mathcal{C}^{(2)}[\tilde{Q}, \tilde{\mathscr{H}}, \tilde{\mathscr{G}}]+O\left(\lambda^{4}\right), \\
\mathscr{G} & =\tilde{\mathscr{G}}+\lambda^{2} \mathcal{D}^{(2)}[\tilde{Q}, \tilde{\mathscr{H}}, \tilde{\mathscr{G}}]+O\left(\lambda^{4}\right),
\end{aligned}
$$


where $\mathcal{A}^{(n)}, \mathcal{B}^{(n)}, \mathcal{C}^{(n)}$, and $\mathcal{D}^{(n)}(n=2,4,6, \ldots)$ are functionals (the odd orders vanish for the Gaussianity).

(iii) Invert Eqs. (A3):

$$
\begin{aligned}
\tilde{Q} & =Q-\lambda^{2} \mathcal{A}^{(2)}[Q, G]+O\left(\lambda^{4}\right), \\
\tilde{G} & =G-\lambda^{2} \mathcal{B}^{(2)}[Q, G]+O\left(\lambda^{4}\right), \\
\tilde{\mathscr{H}} & =\mathscr{H}-\lambda^{2} \mathcal{C}^{(2)}[Q, \mathscr{H}, \mathscr{G}]+O\left(\lambda^{4}\right), \\
\tilde{\mathscr{G}} & =\mathscr{G}-\lambda^{2} \mathcal{D}^{(2)}[Q, \mathscr{H}, \mathscr{G}]+O\left(\lambda^{4}\right) .
\end{aligned}
$$

(iv) By substituting Eqs. (A4) into Eq. (A2), $J$ can be expressed in terms of the representatives $Q, G, \mathscr{H}$, and $\mathscr{G}$ :

$$
J=\mathcal{J}^{(0)}[Q, G, \mathscr{H}, \mathscr{G}]+\lambda \mathcal{J}^{(1)}[Q, G, \mathscr{H}, \mathscr{G}]+O\left(\lambda^{2}\right),
$$

(v) Truncate the renormalized expansion (A5) at the lowest order.

For instance, if we write the exact dynamical equation of $\mathscr{H}$ as $\partial_{t} \mathscr{H}=\lambda I$, applying (i)-(v) to $I$ yields

$$
I=\lambda \mathcal{I}^{(1)}[Q, \mathscr{H}, \mathscr{G}]+O\left(\lambda^{2}\right) \stackrel{\text { LRA }}{\approx} \lambda \mathcal{I}^{(1)}[Q \mathscr{H}, \mathscr{G}],
$$

which yields

$$
\partial_{t} \mathscr{H}=\lambda^{2} \mathcal{I}^{(1)}[Q, \mathscr{H}, \mathscr{G}]
$$

Applying the same steps to the other representatives, we obtain a closed set of equations for $Q, G, \mathscr{H}$, and $\mathscr{G}$.

Let us introduce the Fourier transform of the correlation functions and response functions as $H_{i j l m}\left(\mathbf{k} ; t, t^{\prime}\right)(\equiv$ $\left.\mathcal{F}_{\mathbf{r}}^{\mathbf{k}} \mathscr{H}_{i j l m}\left(\mathbf{r} ; t, t^{\prime}\right)\right), G_{i j l m}\left(\mathbf{k} ; t, t^{\prime}\right)\left(\equiv \mathcal{F}_{\mathbf{r}}^{\mathbf{k}} \mathscr{G}_{i j l m}\left(\mathbf{r} ; t, t^{\prime}\right)\right)$,

and $\quad Q_{i j}\left(\mathbf{k} ; t, t^{\prime}\right)\left(\equiv \mathcal{F}_{\mathbf{r}}^{\mathbf{k}}\left\langle u_{i}\left(\mathbf{x}+\mathbf{r}, t^{\prime} \mid t\right) u_{j}\left(\mathbf{x}, t^{\prime}\right)\right\rangle\right)$, where $\mathcal{F}_{\mathbf{r}}^{\mathbf{k}} f(\mathbf{r})\left(\equiv(2 \pi)^{-3} \int d \mathbf{r} \mathrm{e}^{-i \mathbf{k} \cdot \mathbf{r}} f(\mathbf{r})\right)$ represents the Fourier transformation of an arbitrary physical-space function $f(\mathbf{r})$. We also introduce the contracted spectral functions $H\left(k ; t, t^{\prime}\right) \equiv(3 / 2) H_{i j i j}\left(\mathbf{k} ; t, t^{\prime}\right), G_{H}\left(k ; t, t^{\prime}\right) \equiv G_{i j i j}\left(\mathbf{k} ; t, t^{\prime}\right)$, and $Q\left(k ; t, t^{\prime}\right) \equiv(1 / 2)\left(\delta_{i j}-k_{i} k_{j} / k^{2}\right) Q_{i j}\left(\mathbf{k} ; t, t^{\prime}\right)$. Then, for the statistically isotropic case, the closure equations for particle statistics in Fourier space read

$$
\begin{aligned}
\partial_{t} H(k ; t, t)= & 2 \pi \iint_{I_{k}} d p d q k p q\left(1-y^{2}\right) \int_{-\infty}^{t} d s Q(q ; s, t)\left\{H(p ; s, t) G_{H}(k ; s, t)-H(k ; s, t) G_{H}(p ; s, t)\right\} \\
& +\tau_{p}^{2} \frac{k^{2}}{4 \pi} \int_{-\infty}^{t} d s G_{H}(k ; t, s) E_{p}(k ; t, s) \\
\partial_{t} H\left(k ; t, t^{\prime}\right)= & -\frac{3}{2} \pi \iint_{I_{k}} d p d q k p q\left(1-y^{2}\right)\left(1-z^{2}\right) \int_{t^{\prime}}^{t} d s Q(p ; t, s) H\left(k ; t, t^{\prime}\right) \\
& -\frac{3}{2} \pi \iint_{I_{k}} d p d q k p q\left(1-y^{2}\right)\left(1-z^{2}\right) \int_{-\infty}^{t} d s Q(p ; t, s) G_{H}(q ; t, s) H\left(k ; t^{\prime}, s\right) \\
& +\frac{3}{2} \pi \iint_{I_{k}} d p d q k p q\left(1-y^{2}\right)\left(1-z^{2}\right) \int_{-\infty}^{t^{\prime}} d s Q(p ; t, s) H(q ; t, s) G_{H}\left(k ; t^{\prime}, s\right) \\
& +\tau_{p}^{2} \frac{k^{2}}{8 \pi} \int_{-\infty}^{t^{\prime}} d s G_{H}\left(k ; t^{\prime}, s\right) E_{p}(k ; t, s), \\
\partial_{t} G_{H}\left(k ; t, t^{\prime}\right)= & -\frac{3}{2} \pi \iint_{I_{k}} d p d q k p q\left(1-y^{2}\right)\left(1-z^{2}\right) \int_{t^{\prime}}^{t} d s Q(p ; t, s)\left\{G_{H}\left(k ; t, t^{\prime}\right)+G_{H}(q ; t, s) G_{H}\left(k ; s, t^{\prime}\right)\right\},
\end{aligned}
$$

supplemented with the geometrical properties $y \equiv$ $\left(q^{2}+k^{2}-p^{2}\right) /(2 k q), z \equiv\left(p^{2}+k^{2}-q^{2}\right) /(2 k p)$, and $I_{k} \equiv$ $\{(p, q)|| k-p \mid \leqslant q \leqslant k+p\}$. The above set of Eqs. (A8)(A10) and Eq. (2.48) in Ref. [19] provide the complete description of particles and turbulence in terms of secondorder correlations. Equation (A8) is expressed as Eqs. (9)(12) in Sec. IV. The number-density spectrum and PCF are given respectively by $E_{\theta}(k, t)=4 \pi k^{2} H(k ; t, t)$ and $\Theta(r, t)=$ $4 \pi \int_{0}^{\infty} k r^{-1} \sin (k r) H(k ; t, t) d k$.

On the choice of the representative variable $\mathscr{H}_{i j}$, one may recall the strain-based closures of Ref. [24,33], where the derivative of the velocity field appears as the representative variable. Whereas, being similar to each other, there is an essential gap between strain-based and Hessian-based closures; strain-based closures attempt to eliminate the fluid's time scale under random rotation, while $H$-based LRA avoids the long-time memory of the scalar field. Also $H$-based LRA is mathematically more feasible than these strain-based theories, because we can reuse the results from the original LRA for velocity statistics in $H$-based LRA, whereas a complex reformulation of the velocity-statistics closure is required in the strain-based closure.
[1] R. A. Shaw, Annu. Rev. Fluid Mech. 35, 183 (2003).

[2] C. W. Ormel, M. Spaans, and A. G. G. M. Tielens, Astron. Astrophys. 461, 215 (2007).

[3] J. Blum and G. Wurm, Annu. Rev. Astron. Astrophys. 46, 21 (2008).
[4] M. R. Maxey, J. Fluid Mech. 174, 441 (1987).

[5] K. D. Squires and J. K. Eaton, Phys. Fluids 3, 1169 (1991).

[6] A. N. Kolmogorov, Dokl. Akad. Nauk SSSR 30, 299 (1941) (reprinted in Proc. R. Soc. Lond. A 434, 9 (1991)). 
[7] T. Elperin, N. Kleeorin, and I. Rogachevskii, Phys. Rev. E 58, 3113 (1998).

[8] E. Balkovsky, G. Falkovich, and A. Fouxon, Phys. Rev. Lett. 86, 2790 (2001).

[9] A. D. Bragg, P. J. Ireland, and L. R. Collins, Phys. Rev. E 92, 023029 (2015).

[10] L. I. Zaichik and V. M. Alipchenkov, Phys. Fluids 19, 113308 (2007).

[11] H. Yoshimoto and S. Goto, J. Fluid Mech. 577, 275 (2007).

[12] S. Goto and J. C. Vassilicos, Phys. Fluids 18, 115103 (2006).

[13] K. Matsuda, R. Onishi, M. Hirahara, R. Kurose, K. Takahashi, and S. Komori, J. Atmos. Sci. 71, 3569 (2014).

[14] M. Bassenne, J. Urzay, K. Schneider, and P. Moin, Phys. Rev. Fluids 2, 054301 (2017).

[15] E. Saw, R. Shaw, S. Ayyalasomayajula, P. Chuang, and Á. Gylfason, Phys. Rev. Lett. 100, 214501 (2008).

[16] M. R. Maxey and J. J. Riley, Phys. Fluids 26, 883 (1983).

[17] J. Bec, M. Cencini, and R. Hillerbrand, Phys. Rev. E 75, 025301(R) (2007).

[18] J. Bec, L. Biferale, M. Cencini, A. Lanotte, S. Musacchio, and F. Toschi, Phys. Rev. Lett. 98, 084502 (2007).
[19] Y. Kaneda, J. Fluid Mech. 107, 131 (1981).

[20] Y. Kaneda, Phys. Fluids 29, 701 (1986).

[21] Z. Warhaft, Annu. Rev. Fluid Mech. 32, 203 (2000).

[22] T. Gotoh and P. K. Yeung, Passive Scalar Transport in Turbulence: A Computational Perspective, in Ten Chapters in Turbulence, edited by P. A. Davidson, Y. Kaneda, and K. R. Sreenivasan (Cambridge University Press, Cambridge, 2013), pp. 87-131.

[23] T. Gotoh and T. Watanabe, Phys. Rev. Lett. 115, 114502 (2015).

[24] T. Gotoh, J. Nagaki, and Y. Kaneda, Phys. Fluids 12, 155 (2000).

[25] K. Matsuda, R. Onishi, R. Kurose, and S. Komori, Phys. Rev. Lett. 108, 224502 (2012).

[26] G. Falkovich, Phys. Fluids 6, 1411 (1994).

[27] Y. Kaneda, T. Ishihara, M. Yokokawa, K. Itakura, and A. Uno, Phys. Fluids 15, L21 (2003).

[28] T. Gotoh and D. Fukayama, Phys. Rev. Lett. 86, 3775 (2001).

[29] Y. Tsuji and T. Ishihara, Phys. Rev. E 68, 026309 (2003).

[30] A. N. Kolmogorov, J. Fluid Mech. 13, 82 (1962).

[31] L. Wang, A. Wexler, and Y. Zhou, J. Fluid Mech. 415, 117 (2000).

[32] Y. Zhou, A. Wexler, and L. Wang, Phys. Fluids 10, 1206 (1998).

[33] R. H. Kraichnan and J. R. Herring, J. Fluid Mech. 88, 355 (1978). 